\title{
SUMS OF AN ENTIRE FUNCTION IN CERTAIN WEIGHTED $L^{2}$-SPACES
}

\author{
BRuno BRIVE
}

\begin{abstract}
We consider the functional equation $f(z+\sigma)-f(z)=g(z)$ where $\sigma$ is a complex number, $f$ and $g$ are entire functions of a complex variable $z$, with growth conditions. We prove the existence of certain types of solutions of this equation by an a priori estimate method in certain weighted $L^{2}$-spaces.
\end{abstract}

\section{Introduction}

We consider the functional equation

$$
f(z+\sigma)-f(z)=g(z)
$$

where $\sigma$ is a non zero complex number, $g$ a given function and $f$ an unknown function, both of one complex variable $z$.

If (1.1) occurs when $\sigma=1, \mathrm{f}$ is usually said to be the sum of $g$. This is an old question. In 1887, Guichard [5] and later in 1897, Hurwitz [7] proved that any given entire function admits an entire sum. A modern treatment of this subject can be found in $[\mathbf{1}]$.

One can formulate the following more precise problem:

(1.2) Let $\sigma \in \mathbf{C}^{*}$. Given $s>0, \alpha>0$ and an entire function $g$ satisfying the growth condition

$$
g(z)=O\left(e^{s|z|^{\alpha}}\right)
$$

as $|z| \rightarrow+\infty$, does there exist an entire function $f$ satisfying (1.1) and an analogous growth condition

$$
f(z)=O\left(e^{t|z|^{\beta}}\right)
$$

2000 Mathematics Subject Classification. Primary: 30D05; Secondary: 30D15, 39A70, 39B32.

Key words. Entire functions, Bergman spaces, difference operators, translation operators, a priori estimates. 
as $|z| \rightarrow+\infty$, with precise relations on the relative values of $\alpha, \beta, s, t$ and $\sigma$ ?

In 1935, Whittaker [16] showed that any entire function of given order $\alpha$ admits an entire sum of the same order.

We consider the above question from the point of view of functional analysis.

Let $\mathcal{O}(\mathbf{C})$ be the set of all entire functions $f: \mathbf{C} \rightarrow \mathbf{C}$, equipped with the topology of uniform convergence on compact subsets of $\mathbf{C}$. For $\sigma \in \mathbf{C}$, we denote by $\tau_{\sigma}$ the translation $z \mapsto z+\sigma$ in $\mathbf{C}$ and by $T_{\sigma}$ the operator $f \mapsto f \circ \tau_{\sigma}$ acting on $f \in \mathcal{O}(\mathbf{C})$. We call $T_{\sigma}$ the translation operator associated to $\sigma$. We also denote by $I$ the identity operator and by $S_{\sigma}$ the operator $T_{\sigma}-I$. If $\sigma=1$, we simply write $\tau$ for $\tau_{1}, T$ for $T_{1}$ and $S$ for $S_{1}$.

We substitute the growth condition (1.3) by the integrability condition

$$
\int_{\mathbf{C}}|g(z)|^{2} e^{-s|z|^{\alpha}} d \lambda(z)<+\infty
$$

where $d \lambda$ denotes the Lebesgue measure on $\mathbf{C}$. Entire functions satisfying (1.5) form a Hilbert space that we shall denote by $H(s, \alpha)$. The $H(s, \alpha)$ spaces are a particular case of general weighted $L^{p}$-spaces: Given a domain $\Omega$ in $\mathbf{C}$, a number $p$ in $] 1,+\infty[$ and a measurable function $\varphi: \Omega \rightarrow \mathbf{R}$, the space $L^{p}(\Omega, \varphi)$, is defined to be the set of all measurable functions $g: \Omega \rightarrow \mathbf{C}$ such that

$$
\int_{\Omega}|g|^{p} e^{-\varphi} d \lambda<+\infty
$$

This is a Banach space with the norm $\|f\|=\left(\int_{\Omega}|f|^{p} e^{-\varphi} d \lambda\right)^{\frac{1}{p}}$. When $p=2, L^{2}(\Omega, \varphi)$ is a Hilbert space for the product $\langle f, g\rangle=\int_{\Omega} f \bar{g} e^{-\varphi} d \lambda$.

Let $\mathcal{O}(\Omega)$ be the set of all holomorphic functions on $\Omega$. If $e^{\frac{1}{p-1} \varphi}$ is locally integrable over $\Omega$ then $L^{p}(\Omega, \varphi) \cap \mathcal{O}(\Omega)$ is a closed subspace of $L^{p}(\Omega, \varphi)$, see $[3]$.

We consider the weights $\varphi(z)=s|z|^{\alpha}$, with $s>0$ and $\alpha>0$. For $p \in$ $[1,+\infty[$ and $\alpha=2$ we obtain the Bargmann-Fock spaces (See e.g. [13], [15], [11].) For $p=2$ and $\alpha>0$ then $H(s, \alpha)=L^{2}(\mathbf{C}, \varphi) \cap \mathcal{O}(\mathbf{C})$.

Our results concern the existence of solutions of the equation (1.1), in the $H(s, \alpha)$ spaces, when $\alpha \leqslant 1$.

Let us now state our main theorems. 
Theorem 1. Let $\alpha \leqslant 1$.

(1) For any $\beta>\alpha$ and any $t>0, S$ induces a closed, densely defined and surjective unbounded operator from $H(t, \beta)$ onto $H(1, \alpha)$.

(2) There exists $t_{0}$ such that for any $t>t_{0}, S$ induces a closed, densely defined and surjective unbounded operator from $H(t, \alpha)$ onto $H(1, \alpha)$.

Theorem 2. Let $\alpha \leqslant 1$ and $s>0$.

(1) If $\alpha<1$, there exists $t^{\prime}>0$ such that for any $t>t^{\prime}, S_{\sigma}$ induces a closed, densely defined, surjective unbounded operator from $H(t, \alpha)$ onto $H(s, \alpha)$.

(2) If $s|\sigma|<\sqrt{2 \pi}$, there exists $t^{\prime \prime}>0$ such that for any $t>t^{\prime \prime}, S_{\sigma}$ induces a closed, densely defined, surjective unbounded operator from $H(t, 1)$ onto $H(s, 1)$.

Theorem 1 was announced in [2]. It gives an answer to (1.2) in cases $\alpha \leqslant 1, \sigma=1$ and $s=1$.

In order to prove the surjectivity of the operators in Theorem 1, we use a priori estimates. More precisely, we apply the following statement $[\mathbf{6}]$.

(1.6) Let $H_{1}$ and $H_{2}$ be two Hilbert spaces and $u$ a closed, densely defined unbounded operator from $\mathrm{H}_{1}$ to $\mathrm{H}_{2}$. Then $u$ is surjective if and only if there exists a positive constant $C$ such that for any $g \in \operatorname{dom}\left(u^{*}\right)$

$$
\|g\|_{H_{2}} \leqslant C\left\|u^{*} g\right\|_{H_{1}} .
$$

The $H(s, \alpha)$ spaces enable us to reduce the preceeding inequality to an inequality between series, whose coefficients are computed from the Taylor series of the functions. Our method may apply to more general functional equations than (1.1).

This article is organized as follows.

In Section 2, we present the material of weighted $L^{2}$-spaces of entire functions. In particular, we give the Aronszajn-Bergman reproducing kernel for a general class of such spaces.

In Section 3, we study the action of translation operators on the $H(s, \alpha)$ spaces.

The Sections 4, 5 and 6 are devoted to the proofs of Theorems 1 and 2.

Finally, in Section 7, we give further results. In particular we determine all periodic functions in $H(s, \alpha)$ in the case $\alpha \leqslant 1$ (Subsection 7.2). 
We also give a growth condition for functions belonging in $H(s, \alpha)$ (Subsection 7.1). This enables us to state a theorem in the spirit of Whittaker's result.

\section{Weighted $L^{2}$-spaces of entire functions}

\subsection{Aronszajn-Bergman Kernel in weighted $L^{2}$-spaces.}

The Bergman kernel theory in $L^{2}$-spaces is well-known [8], [4]. The analogous theory in general weighted $L^{2}$-spaces may also be regarded as a particular case of the Aronszajn-Bergman reproducing kernel theory $[\mathbf{1 7}]$.

Let $\Omega$ be a domain in $\mathbf{C}$ and $\varphi: \Omega \rightarrow \mathbf{R}$ a measurable function on $\Omega$. Recall that if $e^{\varphi}$ is locally integrable on $\Omega$ then $H=L^{2}(\Omega, \varphi) \cap \mathcal{O}(\Omega)$ is a closed subspace of the Hilbert space $L^{2}(\Omega, \varphi)$.

Let $f \in H$. Let $z$ be in $\Omega$ and $r>0$ such that the closed disk of center $z$ and radius $r, \bar{D}=\{w \in \mathbf{C} /|w-z| \leqslant r\}$, is contained in $\Omega$. Being holomorphic, $f$ satisfies the mean-value property

$$
f(z)=\frac{1}{\pi r^{2}} \int_{\bar{D}} f d \lambda .
$$

Writing $f=f e^{-\frac{1}{2} \varphi} e^{\frac{1}{2} \varphi}$ and applying Cauchy-Schwarz inequality in the above integral, we are led to the inequality

$$
|f(z)| \leqslant \frac{1}{\pi r^{2}}\left(\int_{\bar{D}} e^{\varphi} d \lambda\right)^{\frac{1}{2}}\|f\| .
$$

It follows [17] that $H$ has a reproducing kernel $K$, that is a map $K: \Omega \times \Omega \rightarrow \mathbf{C}$ with the properties:

(i) For any fixed $w$ in $\Omega$, the function $K_{w}: z \mapsto K(z, w)$ belongs to $H$.

(ii) For any $f$ in $H$ and $z$ in $\Omega, f(z)=\int_{\Omega} f(w) K(z, w) e^{-\varphi(w)} d \lambda(w)$.

Now, let the operator $P$ be defined on $L^{2}(\Omega, \varphi)$ by

$$
P g(z)=\int_{\Omega} g(w) K(z, w) e^{-\varphi(w)} d \lambda(w)
$$

for any $g$ in $L^{2}(\Omega, \varphi)$ and any $z$ in $\Omega$. Then for any $g \in L^{2}(\Omega, \varphi), P g$ belongs to $H$ and the operator $P: L^{2}(\Omega, \varphi) \rightarrow H$ coincides with the orthogonal projection.

If $\left(\phi_{n}\right)_{n \geqslant 0}$ is a complete orthonormal system of $H$, then $K(z, w)=$ $\sum_{n=0}^{+\infty} \overline{\phi_{n}(w)} \phi_{n}(z)$ for any $z$ and $w$ in $\Omega$. 


\subsection{A particular class of weighted $L^{2}$-spaces.}

We consider the case where $\Omega=\mathbf{C}$ and $\varphi$ depends only on the modulus of the variable.

Proposition 1. Let $\phi: \mathbf{R}_{+} \rightarrow \mathbf{R}$ be measurable and such that for any positive integer $p$, the integral $\int_{0}^{+\infty} r^{p} e^{-\phi(r)} d r$ converges. Let $\varphi: z \mapsto$ $\phi(|z|), z \in \mathbf{C}$, and $H=L^{2}(\mathbf{C}, \varphi) \cap \mathcal{O}(\mathbf{C})$.

(i) The monomials $\gamma_{n}: z \mapsto z^{n}, n \in \mathbf{N}$, form a complete orthogonal system in $H$.

(ii) Let $f(z)=\sum_{n=0}^{+\infty} a_{n} z^{n}$ be an entire function. Then

$$
f \in H \Longleftrightarrow \sum_{n=0}^{+\infty}\left|a_{n}\right|^{2} \int_{0}^{+\infty} r^{2 n+1} e^{-\phi(r)} d r<+\infty .
$$

If $f \in H$, then $\|f\|^{2}=2 \pi \sum_{n=0}^{+\infty}\left|a_{n}\right|^{2} \int_{0}^{+\infty} r^{2 n+1} e^{-\phi(r)} d r$, (the Parseval formula). The Taylor series of $f$ in 0 converges in $L^{2}(\mathbf{C}, \varphi)$.

(iii) The Aronszajn-Bergman reproducing kernel is given in $H$ by

$$
K(z, w)=\sum_{n=0}^{+\infty} \frac{(z \bar{w})^{n}}{\left\|\gamma_{n}\right\|^{2}} .
$$

Proof: For any two integers $p$ and $q$, we have $\left\langle\gamma_{p}, \gamma_{q}\right\rangle=\int_{\mathbf{C}} z^{p} \bar{z}^{q} e^{-\varphi(z)} d \lambda(z)$. Computing this integral with polar coordinates, we find that $\left\langle\gamma_{p}, \gamma_{q}\right\rangle=0$ if $p \neq q$ and $\left\|\gamma_{n}\right\|^{2}=2 \pi \int_{0}^{+\infty} r^{2 n+1} e^{-\phi(r)} d r$. Now, integrating the classical Gutzmer formula

$$
\frac{1}{2 \pi} \int_{0}^{2 \pi}\left|f\left(r e^{i \theta}\right)\right|^{2} d \theta=\sum_{n=0}^{+\infty}\left|a_{n}\right|^{2} r^{2 n}
$$

in $r$ over $\left[0,+\infty\right.$ [, for $f(z)=\sum_{n=0}^{+\infty} a_{n} z^{n}$, we find the Parseval formula for $f$ in $H$.

\subsection{The $H(s, \alpha)$ spaces.}

Let $\alpha>0$ and $s>0$. The Hilbert space $H(s, \alpha)$ is the particular case of the situation described in Proposition 1 where $\phi(r)=s r^{\alpha}$. Here we have

$$
\int_{0}^{+\infty} r^{p} e^{-\phi(r)} d r=\int_{0}^{+\infty} r^{p} e^{-s r^{\alpha}} d r=\frac{1}{\alpha} s^{-\frac{1}{\alpha}(p+1)} \Gamma\left(\frac{p+1}{\alpha}\right)<+\infty .
$$


Throughout this article, we shall denote by:

$$
\begin{aligned}
& \|f\|_{s, \alpha} \quad \text { the norm of any function } f \text { in } H(s, \alpha), \\
& e_{n}(s, \alpha) \text { the function } \frac{\gamma_{n}}{\left\|\gamma_{n}\right\|_{s, \alpha}} \text { for } n \in \mathbf{N} .
\end{aligned}
$$

Proposition 1 states that $\left(e_{n}(s, \alpha)\right)_{n \in \mathbf{N}}$ is a basis of $H(s, \alpha)$. Let $f \in$ $H(s, \alpha)$ with $f(z)=\sum_{n=0}^{+\infty} a_{n} z^{n}$ its Taylor expansion at the origin. Then $f=\sum_{n=0}^{+\infty} a_{n}\left\|\gamma_{n}\right\|_{s, \alpha} e_{n}(s, \alpha)$ is the expansion of $f$ in the basis $\left(e_{n}(s, \alpha)\right)_{n \in \mathbf{N}}$ in $H(s, \alpha)$. This gives the Parseval formula

$$
\|f\|_{s, \alpha}^{2}=\sum_{n=0}^{+\infty}\left|a_{n}\right|^{2}\left\|\gamma_{n}\right\|_{s, \alpha}^{2}
$$

where $\left\|\gamma_{n}\right\|_{s, \alpha}^{2}=\frac{2 \pi}{\alpha} s^{-\frac{2}{\alpha}(n+1)} \Gamma\left(\frac{2}{\alpha}(n+1)\right)$. The Aronszajn-Bergman reproducing kernel is therefore given in $H(s, \alpha)$ by

$$
K(z, w)=\sum_{n=0}^{+\infty} \frac{(z \bar{w})^{n}}{\frac{2 \pi}{\alpha} s^{-\frac{2(n+1)}{\alpha}} \Gamma\left(\frac{2(n+1)}{\alpha}\right)} .
$$

Note that if moreover $\alpha=2$, then

$$
K(z, w)=\frac{s}{\pi} e^{s z \bar{w}},
$$

as it is well-known (see [15]).

The next proposition compares the two spaces $H(s, \alpha)$ and $H(t, \beta)$. See also $[\mathbf{1 1}]$.

Proposition 2. Let $\alpha>0$ and $s>0$.

(i) For any $\beta>\alpha$ and any $t>0, H(s, \alpha) \subset H(t, \beta)$.

(ii) For any $t>s, H(s, \alpha) \subset H(t, \alpha)$.

The inclusions are strict and dense.

Proof: The inclusions are clear. A polynomial is always contained in $H(s, \alpha)$ thus $H(s, \alpha)$ is a dense subspace of $H(t, \beta)$ when $H(s, \alpha) \subset$ $H(t, \beta)$.

Set $a_{n}=s^{\frac{1}{\alpha}(n+1)} \Gamma\left(\frac{2}{\alpha}(n+1)\right)^{-\frac{1}{2}}, n \in \mathbf{N}$ and $f(z)=\sum_{n=0}^{+\infty} a_{n} z^{n}$. Then $f \notin H(s, \alpha)$, but

1. $f \in H(t, \beta)$ if $\beta>\alpha$ and $t>0$.

2. $f \in H(t, \alpha)$ if $t>s$. 


\section{Translation operators}

Let $\sigma \in \mathbf{C}$ and $T_{\sigma}$ be the corresponding translation operator. For $s>0, \alpha>0, t>0$ and $\beta>0, T_{\sigma}$ induces an unbounded operator from $H(t, \beta)$ to $H(s, \alpha)$ with domain

$$
\operatorname{dom}\left(T_{\sigma}\right)=\left\{f \in H(t, \beta) \mid T_{\sigma} f \in H(s, \alpha)\right\} .
$$

It is not surprising that the properties of $T_{\sigma}$ depend on the relative sizes of the spaces $H(t, \beta)$ and $H(s, \alpha)$. We explain this idea more precisely in the two following propositions.

Proposition 3. Let $\alpha>0$ and $s>0$. Let $\sigma \in \mathbf{C}^{*}$.

(i) For any $\beta>\alpha$ and any $t>0, T_{\sigma}$ induces a bounded operator from $H(s, \alpha)$ to $H(t, \beta)$.

(ii) For any $t>s, T_{\sigma}$ induces a bounded operator from $H(s, \alpha)$ to $H(t, \alpha)$.

(iii) If $\alpha \leqslant 1$ then $T_{\sigma}$ induces a one-to-one bounded operator on $H(s, \alpha)$.

For a more general statement concerning $L^{p}$-spaces and radial weights, see $[3]$.

Proposition 4. Let $\alpha>0$ and $s>0$. Let $\sigma \in \mathbf{C}^{*}$.

(i) For any $\beta>\alpha$ and any $t>0, T_{\sigma}$ induces a closed, densely defined unbounded operator from $H(t, \beta)$ to $H(s, \alpha)$.

(ii) For any $t>s, T_{\sigma}$ induces a closed, densely defined unbounded operator from $H(t, \alpha)$ to $H(s, \alpha)$.

(iii) If $\alpha>1$ then $T_{\sigma}$ induces a closed, densely defined unbounded operator in $H(s, \alpha)$.

In each of cases (i), (ii) and (iii), the domain of the operator is not closed.

Proof of Proposition 3: Let $\beta>0$ and $t>0$. For any $f$ in $H(s, \alpha)$ we have the estimate

$$
\left\|T_{\sigma} f\right\|_{t, \beta}^{2} \leqslant \sup _{z \in \mathbf{C}} e^{s|z+\sigma|^{\alpha}-t|z|^{\beta}} \cdot\|f\|_{s, \alpha}^{2} .
$$

The proposition is therefore a consequence of the following observations. 
(i) If $\beta>\alpha$ and $t>0$ then the function $z \mapsto e^{s|z+\sigma|^{\alpha}-t|z|^{\beta}}$ vanishes at infinity, in particular it is bounded over $\mathbf{C}$.

(ii) If $t>s$ then the function $z \mapsto e^{s|z+\sigma|^{\alpha}-t|z|^{\alpha}}$ vanishes at infinity, in particular it is bounded over $\mathbf{C}$.

(iii) The function $z \mapsto e^{s|z+\sigma|^{\alpha}-s|z|^{\alpha}}$ is bounded over $\mathbf{C}$ if and only if $\alpha \leqslant 1$.

Proof of Proposition 4: The operator is always densely defined because its domain always contains polynomials. Consider case (i). Let us prove that $T_{\sigma}$ is closed, that is, its graph $\left\{\left(f, T_{\sigma} f\right) \mid f \in \operatorname{dom}\left(T_{\sigma}\right)\right\}$ is closed in $H(t, \beta) \times H(s, \alpha)$. Consider a sequence $\left(f_{n}, T_{\sigma} f_{n}\right)_{n \geqslant 0}$ in this graph, converging to $(f, g)$ in $H(t, \beta) \times H(s, \alpha)$. We will prove that $f \in \operatorname{dom}\left(T_{\sigma}\right)$ and that $g=T_{\sigma} f$. By our hypothesis the sequence $\left(f_{n}\right)_{n \geqslant 0}$ converges to $f$ in $H(t, \beta)$, hence also in $\mathcal{O}(\mathbf{C})$, that is, uniformly on compact subsets of $\mathbf{C}$. Thus $\lim _{n \rightarrow+\infty} f_{n} \circ \tau_{\sigma}=f \circ \tau_{\sigma}$ in $\mathcal{O}(\mathbf{C})$. But $f_{n} \circ \tau_{\sigma}=T_{\sigma} f_{n}$ and by hypothesis, the sequence $\left(T_{\sigma} f_{n}\right)_{n \geqslant 0}$ converges to $g$ in $H(s, \alpha)$, hence also in $\mathcal{O}(\mathbf{C})$. Unicity of the limit in $\mathcal{O}(\mathbf{C})$ shows that $g=f \circ \tau_{\sigma}$, and since $g \in H(s, \alpha)$ this proves that $f \in \operatorname{dom}\left(T_{\sigma}\right)$ and that $g=T_{\sigma} f$. Therefore $T_{\sigma}$ is closed in case (i). The same proof holds for the corresponding assertions in cases (ii) and (iii).

We now look at the domain of the operator $T_{\sigma}$. Let $\beta>0$ and $s>0$. We study $\sup _{n \in \mathbf{N}}\left\|T_{\sigma} e_{n}(t, \beta)\right\|_{s, \alpha}$. We have

$$
\left\|T_{\sigma} e_{n}(t, \beta)\right\|_{s, \alpha}^{2}=\frac{1}{\left\|\gamma_{n}\right\|_{t, \beta}^{2}} \int_{\mathbf{C}}|z+\sigma|^{2 n} e^{-s|z|^{\alpha}} d \lambda(z) .
$$

Set $z=\sigma w$ in the above integral, this gives

$$
\begin{aligned}
\left\|\gamma_{n}\right\|_{t, \beta}^{2} \cdot\left\|T_{\sigma} e_{n}(t, \beta)\right\|_{s, \alpha}^{2} & =|\sigma|^{2 n+2} \int_{\mathbf{C}}|w+1|^{2 n} e^{-s|\sigma|^{\alpha}|w|^{\alpha}} d \lambda(w) \\
& =|\sigma|^{2 n+2} \int_{-\pi}^{+\pi} d \theta \int_{0}^{+\infty}\left(r^{2}+2 r \cos \theta+1\right)^{n} e^{-s|\sigma|^{\alpha} r^{\alpha}} r d r .
\end{aligned}
$$

By considering only the values of $\theta$ such that $\cos \theta \geqslant 0$, we obtain

$$
\left\|\gamma_{n}\right\|_{t, \beta}^{2} \cdot\left\|T_{\sigma} e_{n}(t, \beta)\right\|_{s, \alpha}^{2} \geqslant|\sigma|^{2 n+2} \int_{-\frac{\pi}{2}}^{+\frac{\pi}{2}} d \theta \int_{0}^{+\infty}\left(r^{2}+1\right)^{n} e^{-s|\sigma|^{\alpha} r^{\alpha}} r d r .
$$


Since $\left(r^{2}+1\right)^{n} \geqslant r^{2 n}+n r^{2 n-1}$, the right-hand side of the above inequality is bounded below by

$$
\pi|\sigma|^{2 n+2}\left(\int_{0}^{+\infty} r^{2 n+1} e^{-s|\sigma|^{\alpha} r^{\alpha}} r d r+n \int_{0}^{+\infty} r^{2 n} e^{-s|\sigma|^{\alpha} r^{\alpha}} r d r\right)
$$

and so

$$
\left\|T_{\sigma} e_{n}(t, \beta)\right\|_{s, \alpha}^{2} \geqslant \frac{\beta}{2 \alpha} \frac{s^{-\frac{2}{\alpha}(n+1)}}{t^{-\frac{2}{\beta}(n+1)}} \frac{\Gamma\left(\frac{2(n+1)}{\alpha}\right)}{\Gamma\left(\frac{2(n+1)}{\beta}\right)}+\frac{\beta}{2 \alpha}|\sigma| n \frac{s^{-\frac{2 n+1}{\alpha}}}{t^{-\frac{2}{\beta}(n+1)}} \frac{\Gamma\left(\frac{2 n+1}{\alpha}\right)}{\Gamma\left(\frac{2(n+1)}{\beta}\right)} .
$$

We now look at the asymptotic behavior of the right-hand side of the above inequality as $n \rightarrow+\infty$. We consider three cases.

1. If $\beta>\alpha$, the first term behaves like $n^{2\left(\frac{1}{\alpha}-\frac{1}{\beta}\right) n}$ which tends to $+\infty$ as $n \rightarrow+\infty$.

2. If $\beta=\alpha$ and $t>s$, the first term behaves like $\left(\frac{s}{t}\right)^{-\frac{2}{\alpha}(n+1)}$ which tends to $+\infty$ as $n \rightarrow+\infty$.

3. If $\beta=\alpha$ and $t=s$, the above inequality may also be written as

$$
\left\|T_{\sigma} e_{n}(s, \alpha)\right\|_{s, \alpha}^{2} \geqslant \frac{1}{2}\left(1+|\sigma| s^{\frac{1}{\alpha}} \frac{n \Gamma\left(\frac{2 n+1}{\alpha}\right)}{\Gamma\left(\frac{2(n+1)}{\alpha}\right)}\right) .
$$

The second term on the right behaves like $n^{\left(1-\frac{1}{\alpha}\right)}$ which tends to $+\infty$ as $n \rightarrow+\infty$ if $\alpha>1$.

In the above three cases (which correspond respectively to cases (i), (ii) and (iii) of the proposition), we have $\sup _{n \in \mathbf{N}}\left\|T_{\sigma} e_{n}(t, \beta)\right\|_{s, \alpha}=+\infty$, so that an application of the closed-graph theorem shows that the domain of $T_{\sigma}$ is not closed.

Remark. If $\alpha \leqslant 1$ then we may specify the domain of $T_{\sigma}$ in cases (i) and (ii) of Proposition 4. For, if $f$ is such that $T_{\sigma} f$ belongs to $H(s, \alpha)$, $f=T_{-\sigma} T_{\sigma} f \in H(s, \alpha)$ since this space is stable under the action of $T_{-\sigma}$. Hence $\operatorname{dom}\left(T_{\sigma}\right)=H(s, \alpha)$.

\section{Preliminaries to the proofs of Theorems 1 and 2}

Let $\alpha>0, \beta>0, s>0$ and $t>0$. Let

$$
D=\left\{f \in H(t, \beta) \mid S_{\sigma} f \in H(s, \alpha)\right\} .
$$

Then $S_{\sigma}$ induces an unbounded operator from $H(t, \beta)$ to $H(s, \alpha)$ with domain $D$. As in Proposition 4 , one shows that $S_{\sigma}$ is closed and densely defined. 
Proposition 5. Let $g \in \operatorname{dom}\left(S_{\sigma}^{*}\right)$ with $g(z)=\sum_{n=0}^{+\infty} a_{n} z^{n}$. Then $S_{\sigma}^{*} g(z)=$ $\sum_{n=1}^{+\infty} b_{n} z^{n}$ where

$$
b_{n}=\frac{1}{\left\|\gamma_{n}\right\|_{t, \beta}^{2}} \sum_{k=0}^{n-1}\left(\begin{array}{l}
n \\
k
\end{array}\right) \bar{\sigma}^{n-k} a_{k}\left\|\gamma_{k}\right\|_{s, \alpha}^{2} .
$$

Proof: Expanding $S_{\sigma}^{*} g$ in the basis $(e(t, \beta))_{n \geqslant 0}$ of $H(t, \beta)$, we have

$$
S_{\sigma}^{*} g=\sum_{n=0}^{+\infty}\left\langle S_{\sigma}^{*} g, e_{n}(t, \beta)\right\rangle e_{n}(t, \beta)=\sum_{n=0}^{+\infty} \frac{1}{\left\|\gamma_{n}\right\|_{t, \beta}^{2}}\left\langle S_{\sigma}^{*} g, \gamma_{n}\right\rangle \gamma_{n} .
$$

Let $n \geqslant 0$. Since $\gamma_{n} \in \operatorname{dom}\left(S_{\sigma}\right)$, we have

$$
\left\langle S_{\sigma}^{*} g, \gamma_{n}\right\rangle=\left\langle g, S_{\sigma} \gamma_{n}\right\rangle .
$$

Since $S_{\sigma} \gamma_{0}=0$ and $S_{\sigma} \gamma_{n}=\sum_{k=0}^{n-1}\left(\begin{array}{l}n \\ k\end{array}\right) \sigma^{n-k} \gamma_{k}$ if $n \geqslant 1$, we have, for $n \geqslant 1$

$$
\left\langle g, S_{\sigma} \gamma_{n}\right\rangle=\sum_{k=0}^{n-1}\left(\begin{array}{l}
n \\
k
\end{array}\right) \bar{\sigma}^{n-k}\left\langle g, \gamma_{k}\right\rangle
$$

The identity $\left\langle g, \gamma_{k}\right\rangle=a_{k}\left\|\gamma_{k}\right\|_{s, \alpha}^{2}$ thus gives the required result.

We use the notations of Proposition 5. The Parseval formula yields

$$
\|g\|_{s, \alpha}^{2}=\sum_{n=0}^{+\infty}\left|a_{n}\right|^{2}\left\|\gamma_{n}\right\|_{s, \alpha}^{2}
$$

and

$$
\left\|S_{\sigma}^{*} g\right\|_{t, \beta}^{2}=\sum_{n=1}^{+\infty} \frac{|\sigma|^{2 n}}{\left\|\gamma_{n}\right\|_{t, \beta}^{2}}\left|\sum_{k=0}^{n-1}\left(\begin{array}{l}
n \\
k
\end{array}\right) \bar{\sigma}^{-k} a_{k}\left\|\gamma_{k}\right\|_{s, \alpha}^{2}\right|^{2}
$$

If we set

$$
x_{n}=\bar{\sigma}^{-n} a_{n}\left\|\gamma_{n}\right\|_{s, \alpha}^{2}
$$

for $n \geqslant 0$ and

$$
y_{n}=\sum_{k=0}^{n-1}\left(\begin{array}{l}
n \\
k
\end{array}\right) x_{k}
$$


for $n \geqslant 1$, then (4.1) may be written as

$$
\|g\|_{s, \alpha}^{2}=\sum_{n=0}^{+\infty} \frac{\left|x_{n}\right|^{2}}{|\sigma|^{-2 n}\left\|\gamma_{n}\right\|_{s, \alpha}^{2}}
$$

and (4.2) as

$$
\left\|S_{\sigma}^{*} g\right\|_{t, \beta}^{2}=\sum_{n=1}^{+\infty} \frac{\left|y_{n}\right|^{2}}{|\sigma|^{-2 n}\left\|\gamma_{n}\right\|_{t, \beta}^{2}} .
$$

Now, consider the two following assertions:

First assertion. There exists a constant $C>0$ such that the inequality

$$
\|g\|_{s, \alpha} \leqslant C\left\|S_{\sigma}^{*} g\right\|_{t, \beta}
$$

holds for any $g$ in $\operatorname{dom}\left(S_{\sigma}^{*}\right)$.

Second assertion. There exists a constant $C>0$ such that the inequality

$$
\sum_{n=0}^{+\infty} \frac{\left|x_{n}\right|^{2}}{|\sigma|^{-2 n}\left\|\gamma_{n}\right\|_{s, \alpha}^{2}} \leqslant C \sum_{n=1}^{+\infty} \frac{\left|y_{n}\right|^{2}}{|\sigma|^{-2 n}\left\|\gamma_{n}\right\|_{t, \beta}^{2}}
$$

holds for any sequences of complex numbers $\left(x_{n}\right)_{n \geqslant 0}$ and $\left(y_{n}\right)_{n \geqslant 1}$ such that

$$
y_{n}=\sum_{k=0}^{n-1}\left(\begin{array}{l}
n \\
k
\end{array}\right) x_{k} \quad \text { for } n \geqslant 1,
$$

and that the above series are convergent.

It is clear that the first assertion follows from the second one. Actually we shall give sufficient conditions on the values of $\alpha, \beta, s, t$ and $\sigma$ for the second assertion to be valid. Under these conditions, the first assertion will be valid too and, according to (1.6), this will prove that $S_{\sigma}$ is surjective.

In the sequel, our main tools will be:

- The classical properties of the Gamma function and the Beta function, in particular the Stirling formula.

- The asymptotic behavior of Bernoulli numbers.

- The following inequality, which we obtain as a consequence of the log-convexity of the Gamma function: if $a, b, \delta$ and $\theta$ are real $>0$ such that $a \geqslant \theta^{-1}$ and $b \geqslant \theta^{-1}$ then

$$
\frac{\Gamma(a+b)^{\delta}}{\Gamma(\theta(a+b))} \leqslant \frac{\Gamma(2 a)^{\frac{\delta}{2}}}{(\theta a)^{\frac{1}{2}} \Gamma(\theta a)} \cdot \frac{\Gamma(2 b)^{\frac{\delta}{2}}}{(\theta b)^{\frac{1}{2}} \Gamma(\theta b)} .
$$




\section{Proof of Theorem 1}

We now prove the second assertion, with $s=1$ and $\sigma=1$.

\subsection{A triple sum.}

Let $\beta$ and $t$ be two positive numbers. Let $x=\left(x_{n}\right)_{n \geqslant 0}$ and $y=$ $\left(y_{n}\right)_{n \geqslant 1}$ be any two sequences of complex numbers such that

- $y_{n}=\sum_{k=0}^{n-1}\left(\begin{array}{l}n \\ k\end{array}\right) x_{k}$ for $n \geqslant 1$.

- The series $\sum_{n=0}^{+\infty} \frac{\left|x_{n}\right|^{2}}{\left\|\gamma_{n}\right\|_{1, \alpha}^{2}}$ and $\sum_{n=1}^{+\infty} \frac{\left|y_{n}\right|^{2}}{\left\|\gamma_{n}\right\|_{t, \beta}^{2}}$ are convergent.

We first invert the relations between the terms of the sequences $x$ and $y$.

Proposition 6. Let $\left(x_{n}\right)_{n \geqslant 0}$ and $\left(y_{n}\right)_{n \geqslant 1}$ be two sequences of complex numbers such that for any $n \geqslant 1$,

$$
y_{n}=\sum_{k=0}^{n-1}\left(\begin{array}{l}
n \\
k
\end{array}\right) x_{k} .
$$

Then for any $n \geqslant 0$,

$$
x_{n}=\sum_{p=1}^{n+1} \lambda_{p, n} y_{p}
$$

with

$$
\lambda_{p, n}=\frac{n ! B_{n+1-p}}{p !(n+1-p) !} \text { for } 1 \leqslant p \leqslant n+1,
$$

where the $B_{m}, m \geqslant 0$, are the Bernoulli numbers, defined by the expansion

$$
\frac{t}{e^{t}-1}=\sum_{m \geqslant 0} B_{m} \frac{t^{m}}{m !} \text {. }
$$

In particular, $B_{0}=1, B_{1}=-\frac{1}{2}, B_{2}=\frac{1}{6}$ and $B_{m}=0$ for any odd $m>1$.

Proof: The result follows from the identity

$$
\frac{(-1)^{n} B_{n}}{n !}=\left|\begin{array}{cccccc}
\frac{1}{2 !} & 1 & 0 & \ldots & \ldots & 0 \\
\frac{1}{3 !} & \frac{1}{2 !} & 1 & 0 & \ldots & 0 \\
\ldots \ldots & \ldots & \ldots & \ldots \ldots & \ldots \\
\frac{1}{(n+1) !} & \frac{1}{n !} & \ldots & \ldots & \ldots & \frac{1}{2 !}
\end{array}\right|
$$

which can be found in [12, p. 139]. 
According to Proposition 6, we have

$$
\left|x_{n}\right|^{2}=x_{n} \bar{x}_{n}=\sum_{(p, q) \in\{1, \ldots, n+1\}^{2}} \lambda_{p, n} \lambda_{q, n} y_{p} \bar{y}_{q} .
$$

We set

$$
\begin{aligned}
& \Omega=\bigcup_{n \geqslant 0}\{n\} \times\{1, \ldots, n+1\}^{2} \subset \mathbf{N}^{3}, \\
& X(n, p, q)=\frac{\left|\lambda_{p, n} \lambda_{q, n}\right|\left|y_{p} \| y_{q}\right|}{\left\|\gamma_{n}\right\|_{1, \alpha}^{2}} \quad \text { for }(n, p, q) \in \Omega, \\
& \mathfrak{S}=\sum_{(n, p, q) \in \Omega} X(n, p, q) .
\end{aligned}
$$

Then the triangular inequality gives

$$
\sum_{n=0}^{+\infty} \frac{\left|x_{n}\right|^{2}}{\left\|\gamma_{n}\right\|_{1, \alpha}^{2}} \leqslant \mathfrak{S} .
$$

If $(n, p, q) \in \Omega$ then $(n, q, p) \in \Omega$ and $X(n, p, q)=X(n, q, p)$. In other words, the domain $\Omega$ is symmetric with respect to the plane $\{p=q\}$ in $\mathbf{N}^{3}$. For any $k$ the sets $\Omega \cap\{p=q+k\}$ and $\Omega \cap\{p=q-k\}$ are images of each other by this symmetry and the corresponding terms $X(n, p, q)$ contribute equally to the sum $\mathfrak{S}$.

We set

$$
\mathfrak{S}_{(k)}=\sum_{(n, p, q) \in \Omega \cap\{p=q+k\}} X(n, p, q)
$$

for any $k \geqslant 0$. Therefore

$$
\mathfrak{S}=\mathfrak{S}_{(0)}+2 \sum_{k=1}^{+\infty} \mathfrak{S}_{(k)} .
$$

The term $X(n, p, q)$ vanishes when $\lambda_{p, n}$ or $\lambda_{q, n}$ does. Define $\Omega^{\prime}$ to be the set of $(n, p, q)$ in $\Omega$ such that the product $\lambda_{p, n} \lambda_{q, n} \neq 0$. For any $k$, we shall give a geometric representation of points of $\Omega^{\prime} \cap\{p=q+k\}$ : we picture the points of $\Omega \cap\{p=q+k\}$ featuring the elements of $\Omega^{\prime}$ with a $\operatorname{dot}(\bullet)$ and the points of $\Omega \backslash \Omega^{\prime}$ with a cross $(\times)$. 

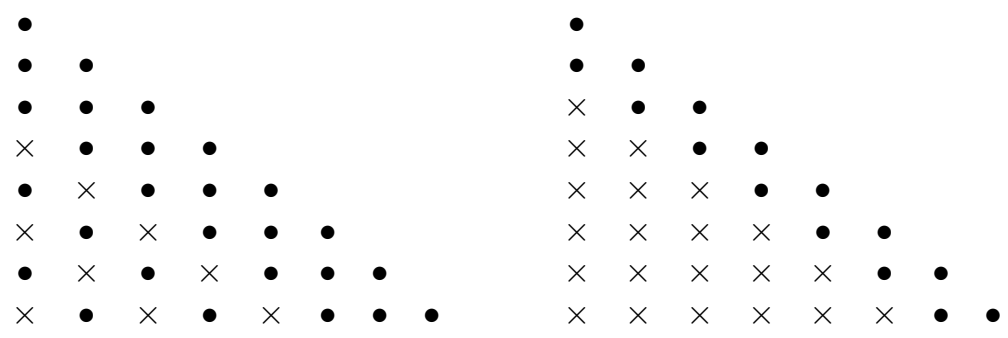

$$
\Omega \cap\{p=q\}
$$

$$
\Omega \cap\{p=q+1\}
$$
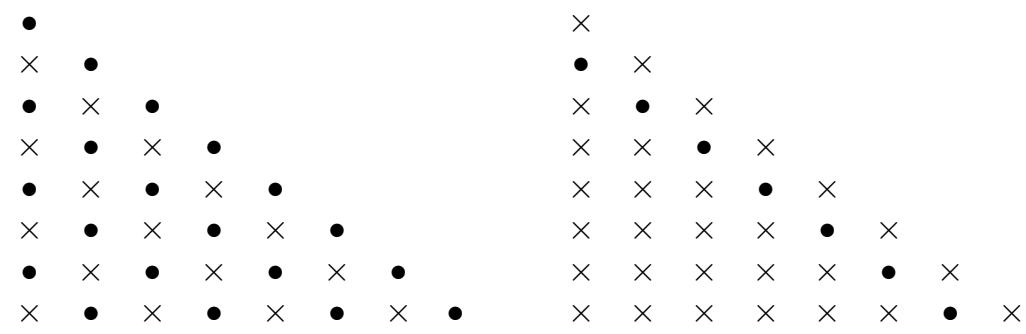

$\Omega \cap\{p=q+k\}$, even $k, k \geqslant 2 \quad \Omega \cap\{p=q+k\}$, odd $k, k \geqslant 3$.

From these pictures we deduce the following expressions:

$$
\mathfrak{S}_{(0)}=\mathfrak{S}_{(0)}^{(1)}+\mathfrak{S}_{(0)}^{(2)}+\mathfrak{S}_{(0)}^{(3)}+\mathfrak{S}_{(0)}^{(4)},
$$

with

$$
\begin{aligned}
& \mathfrak{S}_{(0)}^{(m)}=\sum_{i=0}^{+\infty} a_{(0)}^{(m)}(i)\left|y_{1+i}\right|^{2} \quad \text { for } 1 \leqslant m \leqslant 3, \\
& \mathfrak{S}_{(0)}^{(4)}=\sum_{j=0}^{+\infty} \sum_{i=0}^{+\infty} a_{(0)}^{(4)}(i, j)\left|y_{1+i}\right|^{2},
\end{aligned}
$$

where

$$
\begin{gathered}
a_{(0)}^{(1)}(i)=\frac{\lambda_{1+i, i}^{2}}{\frac{2 \pi}{\alpha} \Gamma\left(\frac{2}{\alpha}(i+1)\right)}, \quad a_{(0)}^{(2)}(i)=\frac{\lambda_{1+i, 1+i}^{2}}{\frac{2 \pi}{\alpha} \Gamma\left(\frac{2}{\alpha}(1+i+1)\right)}, \\
a_{(0)}^{(3)}(i)=\frac{\lambda_{1+i, 2+i}^{2}}{\frac{2 \pi}{\alpha} \Gamma\left(\frac{2}{\alpha}(2+i+1)\right)}, \quad a_{(0)}^{(4)}(i, j)=\frac{\lambda_{1+i, 4+2 j+i}^{2}}{\frac{2 \pi}{\alpha} \Gamma\left(\frac{2}{\alpha}(4+2 j+i+1)\right)}, \\
\mathfrak{S}_{(1)}=\mathfrak{S}_{(1)}^{(1)}+\mathfrak{S}_{(1)}^{(2)},
\end{gathered}
$$


with

$$
\mathfrak{S}_{(1)}^{(m)}=\sum_{i=0}^{+\infty} a_{(1)}^{(m)}(i)\left|y_{2+i}\right|\left|y_{1+i}\right| \quad \text { for } 1 \leqslant m \leqslant 2
$$

where

$$
\begin{aligned}
& a_{(1)}^{(1)}(i)=\frac{\left|\lambda_{2+i, 1+i} \lambda_{1+i, 1+i}\right|}{\frac{2 \pi}{\alpha} \Gamma\left(\frac{2}{\alpha}(1+i+1)\right)}, \\
& a_{(1)}^{(2)}(i)=\frac{\left|\lambda_{2+i, 2+i} \lambda_{1+i, 2+i}\right|}{\frac{2 \pi}{\alpha} \Gamma\left(\frac{2}{\alpha}(2+i+1)\right)},
\end{aligned}
$$

and for $k \geqslant 2$, if $k$ is even

$$
\mathfrak{S}_{(k)}=\sum_{j=0}^{+\infty} \sum_{i=0}^{+\infty} a_{(k)}(i, j)\left|y_{k+1+i}\right|\left|y_{1+i}\right|,
$$

where

$$
a_{(k)}(i, j)=\frac{\left|\lambda_{k+1+i, k+2 j+i} \lambda_{1+i, k+2 j+i}\right|}{\frac{2 \pi}{\alpha} \Gamma\left(\frac{2}{\alpha}(k+2 j+i+1)\right)},
$$

if $k$ is odd

$$
\mathfrak{S}_{(k)}=\sum_{i=0}^{+\infty} a_{(k)}(i)\left|y_{k+1+i}\right|\left|y_{1+i}\right|
$$

where

$$
a_{(k)}(i)=\frac{\left|\lambda_{k+1+i, k+1+i} \lambda_{1+i, k+1+i}\right|}{\frac{2 \pi}{\alpha} \Gamma\left(\frac{2}{\alpha}(k+1+i+1)\right)} .
$$

\subsection{Majorisation of $\mathfrak{S}_{(0)}$.}

The idea of the Majorisation. We have obtained $\mathfrak{S}_{(0)}$ as a sum of four terms that we shall majorise successively. Each one of the first three terms is of the form of a series in one variable $\sum_{i=0}^{+\infty} a(i)\left|y_{1+i}\right|^{2}$. We have an explicit formula for the coefficient $a(i)$. Examinating the asymptotic behavior of the product $a(i)\left\|\gamma_{1+i}\right\|_{t, \beta}^{2}$ as $i \rightarrow+\infty$, we shall prove that explicit conditions on the relative values of $\beta, \alpha$ and $t$ are sufficient for this product to be bounded. Under these conditions, there will therefore exists a positive constant $C$ such that

$$
\sum_{i=0}^{+\infty} a(i)\left|y_{1+i}\right|^{2} \leqslant C \sum_{i=0}^{+\infty} \frac{\left|y_{1+i}\right|^{2}}{\left\|\gamma_{1+i}\right\|_{t, \beta}^{2}} .
$$


The fourth term of $\mathfrak{S}_{(0)}$ is a series in two variables $\sum_{j=0}^{+\infty} \sum_{i=0}^{+\infty} a(i, j)\left|y_{1+i}\right|^{2}$. We still have an explicit formula for $a(i, j)$. We shall prove that explicit conditions on the relative values of $\beta, \alpha$ and $t$, together with the additional condition $\alpha \leqslant 1$ are sufficient for the product $a(i, j)\left\|\gamma_{1+i}\right\|_{t, \beta}^{2}$ to be bounded, uniformly in $i$, by the general term $c(j)$ of a convergent series in $j$. Under these conditions, we shall have

$$
\sum_{j=0}^{+\infty} \sum_{i=0}^{+\infty} a(i, j)\left|y_{1+i}\right|^{2} \leqslant\left(\sum_{j=0}^{+\infty} c(j)\right) \times\left(\sum_{i=0}^{+\infty} \frac{\left|y_{1+i}\right|^{2}}{\left\|\gamma_{1+i}\right\|_{t, \beta}^{2}}\right) .
$$

In the above inequality, the right-hand side has the required form. To obtain the inequality $a(i, j)\left\|\gamma_{1+i}\right\|_{t, \beta}^{2} \leqslant c(j)$, we shall majorise $a(i, j)\left\|\gamma_{1+i}\right\|_{t, \beta}^{2}$ by the product of a function of the single variable $i$ and a function of the single variable $j$, and observe that the function of $i$ is bounded.

The first three terms of $\mathfrak{S}_{(\mathbf{0})}$. Recalling the values of the $\lambda_{p, n}$ (Proposition 6 ), we deduce from the Stirling formula the following asymptotic relations as $i \rightarrow+\infty$ :

$$
\begin{aligned}
& a_{(0)}^{(1)}(i)\left\|\gamma_{1+i}\right\|_{t, \beta}^{2} \sim c \cdot i^{2\left(\frac{1}{\beta}-\frac{1}{\alpha}\right) i} \frac{\left(\frac{2}{\beta}\right)^{\frac{2}{\beta} i}}{\left(\frac{2}{\alpha}\right)^{\frac{2}{\alpha}} i} e^{-2\left(\frac{1}{\beta}-\frac{1}{\alpha}\right) i} t^{-\frac{2}{\beta} i} i^{2\left(\frac{1}{\beta}-\frac{1}{\alpha}\right)+2\left(\frac{1}{\beta}-1\right)}, \\
& a_{(0)}^{(2)}(i)\left\|\gamma_{1+i}\right\|_{t, \beta}^{2} \sim c \cdot i^{2\left(\frac{1}{\beta}-\frac{1}{\alpha}\right) i} \frac{\left(\frac{2}{\beta}\right)^{\frac{2 i}{\beta}}}{\left(\frac{2}{\alpha}\right)^{\frac{2 i}{\alpha}}} e^{-2\left(\frac{1}{\beta}-\frac{1}{\alpha}\right) i} t^{-\frac{2 i}{\beta}} i^{4\left(\frac{1}{\beta}-\frac{1}{\alpha}\right)}, \\
& a_{(0)}^{(3)}(i)\left\|\gamma_{1+i}\right\|_{t, \beta}^{2} \sim c \cdot i^{2\left(\frac{1}{\beta}-\frac{1}{\alpha}\right) i} \frac{\left(\frac{2}{\beta}\right)^{\frac{2 i}{\beta}}}{\left(\frac{2}{\alpha}\right)^{\frac{2 i}{\alpha}}} e^{-2\left(\frac{1}{\beta}-\frac{1}{\alpha}\right) i} t^{-\frac{2 i}{\beta}} i^{4\left(\frac{1}{\beta}-\frac{1}{\alpha}\right)+2\left(1-\frac{1}{\alpha}\right)},
\end{aligned}
$$

where $c$ is a constant independent of $i$. We see that whenever one of the two conditions

(1) $\alpha<\beta$

or

(2) $\alpha=\beta$ and $t>1$

is satisfied then $a_{(0)}^{(1)}(i)\left\|\gamma_{1+i}\right\|_{t, \beta}^{2}, a_{(0)}^{(2)}(i)\left\|\gamma_{1+i}\right\|_{t, \beta}^{2}$ and $a_{(0)}^{(3)}(i)\left\|\gamma_{1+i}\right\|_{t, \beta}^{2}$ are bounded. 
The fourth term of $\mathfrak{S}_{(0)}$. We have

$$
a_{(0)}^{(4)}(i, j)\left\|\gamma_{1+i}\right\|_{t, \beta}^{2}=\frac{(4+2 j+i) !^{2} B_{4+2 j}^{2}}{(1+i) !^{2}(4+2 j) !^{2}} \frac{\frac{2 \pi}{\beta} t^{-\frac{2}{\beta}(i+2)} \Gamma\left(\frac{2}{\beta}(i+2)\right)}{\Gamma\left(\frac{2}{\alpha}(4+2 j+i+1)\right)} .
$$

We deduce that

$$
a_{(0)}^{(4)}(i, j)\left\|\gamma_{1+i}\right\|_{t, \beta}^{2} \leqslant a^{\prime}(j) a^{\prime \prime}(i)
$$

where

$$
\begin{aligned}
& a^{\prime}(j)=\frac{\alpha \Gamma(2(4+2 j))}{\left(\frac{2}{\alpha}(4+2 j)\right)^{1 / 2} \Gamma\left(\frac{2}{\alpha}(4+2 j)\right)} \frac{B_{4+2 j}^{2}}{(4+2 j) !^{2}}, \\
& a^{\prime \prime}(i)=\frac{\Gamma\left(\frac{2}{\beta}(i+2)\right) t^{-\frac{2}{\beta}(i+2)} \Gamma(2(i+1))}{\beta(1+i) !^{2}\left(\frac{2}{\alpha}(i+1)\right)^{1 / 2} \Gamma\left(\frac{2}{\alpha}(i+1)\right)} .
\end{aligned}
$$

We have, as $j \rightarrow+\infty$

$$
a^{\prime}(j) \sim c \cdot j^{4\left(1-\frac{1}{\alpha}\right) j} \frac{4^{4 j}}{\left(\frac{4}{\alpha}\right)^{\frac{4 j}{\alpha}}} e^{-4\left(1-\frac{1}{\alpha}\right) j}(2 \pi)^{-4 j} j^{-\frac{1}{2}+8\left(1-\frac{1}{\alpha}\right)},
$$

and as $i \rightarrow+\infty$

$$
a^{\prime \prime}(i) \sim c \cdot i^{2\left(\frac{1}{\beta}-\frac{1}{\alpha}\right) i} \frac{\left(\frac{2}{\beta}\right)^{\frac{2 i}{\beta}}}{\left(\frac{2}{\alpha}\right)^{\frac{2 i}{\alpha}}} e^{-2\left(\frac{1}{\beta}-\frac{1}{\alpha}\right) i} t^{-\frac{2 i}{\beta}} 2^{2 i} i^{2\left(\frac{1}{\beta}-\frac{1}{\alpha}\right)+2\left(\frac{1}{\beta}-1\right)},
$$

where $c$ denotes some constant independent of $i$ and $j$.

If one of the two following conditions is satisfied

(1) $\alpha \leqslant 1, \alpha<\beta$ and $t>0$

or

(2) $\alpha \leqslant 1, \alpha=\beta$ and $t>2^{\alpha}$

then $a^{\prime}(j)$ is the general term of a convergent series and $a^{\prime \prime}(i)$ is bounded.

\subsection{Majorisation of $\mathfrak{S}_{(1)}$.}

Since for any $i,\left|y_{2+i}\right|\left|y_{1+i}\right| \leqslant\left|y_{2+i}\right|^{2}+\left|y_{1+i}\right|^{2}$, we deduce from (5.2) that $\mathfrak{S}_{(1)}^{(1)} \leqslant \mathfrak{S}_{(1)}^{\left(1^{\prime}\right)}+\mathfrak{S}_{(1)}^{\left(1^{\prime \prime}\right)}$ and $\mathfrak{S}_{(1)}^{(2)} \leqslant \mathfrak{S}_{(1)}^{\left(2^{\prime}\right)}+\mathfrak{S}_{(1)}^{\left(2^{\prime \prime}\right)}$ where

$$
\begin{aligned}
& \mathfrak{S}_{(1)}^{\left(1^{\prime}\right)}=\sum_{i=0}^{+\infty} a_{(1)}^{(1)}(i)\left|y_{2+i}\right|^{2}, \quad \mathfrak{S}_{(1)}^{\left(1^{\prime \prime}\right)}=\sum_{i=0}^{+\infty} a_{(1)}^{(1)}(i)\left|y_{1+i}\right|^{2}, \\
& \mathfrak{S}_{(1)}^{\left(2^{\prime}\right)}=\sum_{i=0}^{+\infty} a_{(1)}^{(2)}(i)\left|y_{2+i}\right|^{2}, \quad \mathfrak{S}_{(1)}^{\left(2^{\prime \prime}\right)}=\sum_{i=0}^{+\infty} a_{(1)}^{(2)}(i)\left|y_{1+i}\right|^{2} .
\end{aligned}
$$


We study each one of the previous sums in the same way as the first three terms of $\mathfrak{S}_{(0)}$. As $i \rightarrow+\infty$, we have the asymptotic relations

$$
\begin{aligned}
& a_{(1)}^{(1)}(i)\left\|\gamma_{2+i}\right\|_{t, \beta}^{2} \sim c \cdot i^{2\left(\frac{1}{\beta}-\frac{1}{\alpha}\right) i} \frac{\left(\frac{2}{\beta}\right)^{\frac{2 i}{\beta}}}{\left(\frac{2}{\alpha}\right)^{\frac{2 i}{\alpha}}} e^{-2\left(\frac{1}{\beta}-\frac{1}{\alpha}\right) i} t^{-\frac{2 i}{\beta}} i^{-1+\frac{6}{\beta}-\frac{4}{\alpha}}, \\
& a_{(1)}^{(1)}(i)\left\|\gamma_{1+i}\right\|_{t, \beta}^{2} \sim c \cdot i^{2\left(\frac{1}{\beta}-\frac{1}{\alpha}\right) i} \frac{\left(\frac{2}{\beta}\right)^{\frac{2 i}{\beta}}}{\left(\frac{2}{\alpha}\right)^{\frac{2 i}{\alpha}}} e^{-2\left(\frac{1}{\beta}-\frac{1}{\alpha}\right) i} t^{-\frac{2 i}{\beta}} i^{-1+4\left(\frac{1}{\beta}-\frac{1}{\alpha}\right)}, \\
& a_{(1)}^{(2)}(i)\left\|\gamma_{2+i}\right\|_{t, \beta}^{2} \sim c \cdot i^{2\left(\frac{1}{\beta}-\frac{1}{\alpha}\right) i} \frac{\left(\frac{2}{\beta}\right)^{\frac{2 i}{\beta}}}{\left(\frac{2}{\alpha}\right)^{\frac{2 i}{\alpha}}} e^{-2\left(\frac{1}{\beta}-\frac{1}{\alpha}\right) i} t^{-\frac{2 i}{\beta}} i^{1+\frac{6}{\beta}-\frac{6}{\alpha}}, \\
& a_{(1)}^{(2)}(i)\left\|\gamma_{1+i}\right\|_{t, \beta}^{2} \sim c \cdot i^{2\left(\frac{1}{\beta}-\frac{1}{\alpha}\right) i} \frac{\left(\frac{2}{\beta}\right)^{\frac{2 i}{\beta}}}{\left(\frac{2}{\alpha}\right)^{\frac{2 i}{\alpha}}} e^{-2\left(\frac{1}{\beta}-\frac{1}{\alpha}\right) i} t^{-\frac{2 i}{\beta}} i^{1+\frac{4}{\beta}-\frac{6}{\alpha}},
\end{aligned}
$$

where $c$ is a constant independent of $i$.

If one of the two conditions

(1) $\alpha<\beta$

or

(2) $\alpha=\beta$ and $t>1$

is satisfied then each one of the previous equivalences is bounded.

5.4. Majorisation of $\sum_{k=2}^{+\infty} \mathfrak{S}_{(k)}$.

Preliminaries. We begin with some computations. Write

$$
\sum_{k=2}^{+\infty} \mathfrak{S}_{(k)}=\sum_{h=1}^{+\infty}\left\{\mathfrak{S}_{(2 h)}+\mathfrak{S}_{(2 h+1)}\right\} .
$$

The term $\mathfrak{S}_{(2 h)}$ is of the form of a double series of positive numbers. We invert the order of the summations and set, for $h \geqslant 1$ and $i \geqslant 0$,

$$
A(h, i)=\sum_{j=0}^{+\infty} \frac{\left|\lambda_{2 h+1+i, 2 h+2 j+i} \lambda_{1+i, 2 h+2 j+i}\right|}{\Gamma\left(\frac{2}{\alpha}(2 h+2 j+i+1)\right)}
$$

and

$$
B(h, i)=\frac{\left|\lambda_{2 h+2+i, 2 h+2+i} \lambda_{1+i, 2 h+2+i}\right|}{\Gamma\left(\frac{2}{\alpha}(2 h+2+i+1)\right)} .
$$


Then

$$
\begin{aligned}
\frac{2 \pi}{\alpha}\left\{\mathfrak{S}_{(2 h)}+\mathfrak{S}_{(2 h+1)}\right\}= & \sum_{i=0}^{+\infty} A(h, i)\left|y_{2 h+1+i}\right|\left|y_{1+i}\right| \\
& +\sum_{i=0}^{+\infty} B(h, i)\left|y_{2 h+2+i}\right|\left|y_{1+i}\right| \\
= & \sum_{i=0}^{+\infty}\left|y_{1+i}\right|\left\{A(h, i)\left|y_{2 h+1+i}\right|+B(h, i)\left|y_{2 h+2+i}\right|\right\} .
\end{aligned}
$$

Let $\left(w_{h}\right)_{h \geqslant 1}$ be a sequence of positive real numbers whose values will be fixed later. For any $i$ the general term of the above sum is equal to $\frac{\left|y_{1+i}\right|}{w_{h}\left\|\gamma_{i+1}\right\|_{t, \beta}}\left\{A(h, i) w_{h}\left\|\gamma_{i+1}\right\|_{t, \beta}\left|y_{2 h+1+i}\right|+B(h, i) w_{h}\left\|\gamma_{i+1}\right\|_{t, \beta}\left|y_{2 h+2+i}\right|\right\}$.

This expression is of the form $c(a+b)$, with

$$
\begin{aligned}
& c=\frac{\left|y_{1+i}\right|}{w_{h}\left\|\gamma_{i+1}\right\|_{t, \beta}}, \\
& a=A(h, i) w_{h}\left\|\gamma_{i+1}\right\|_{t, \beta}\left|y_{2 h+1+i}\right|, \\
& b=B(h, i) w_{h}\left\|\gamma_{i+1}\right\|_{t, \beta}\left|y_{2 h+2+i}\right| .
\end{aligned}
$$

Since the inequality

$$
c(a+b) \leqslant c^{2}+a^{2}+b^{2},
$$

holds for any three positive numbers $a, b$ and $c$, we obtain

$$
\frac{2 \pi}{\alpha} \sum_{h=1}^{+\infty}\left\{\mathfrak{S}_{(2 h)}+\mathfrak{S}_{(2 h+1)}\right\} \leqslant \sum_{h=1}^{+\infty} \sum_{i=0}^{+\infty} \frac{\left|y_{i+1}\right|^{2}}{w_{h}^{2}\left\|\gamma_{i+1}\right\|_{t, \beta}^{2}}+A+B,
$$

where

$$
A=\sum_{h=1}^{+\infty} \sum_{i=0}^{+\infty} A(h, i)^{2} w_{h}^{2}\left\|\gamma_{i+1}\right\|_{t, \beta}^{2}\left|y_{2 h+1+i}\right|^{2}
$$

and

$$
B=\sum_{h=1}^{+\infty} \sum_{i=0}^{+\infty} B(h, i)^{2} w_{h}^{2}\left\|\gamma_{i+1}\right\|_{t, \beta}^{2}\left|y_{2 h+2+i}\right|^{2} .
$$


The idea of the majorisation. The first term in the right member of (5.7) is equal to $\left(\sum_{h=1}^{+\infty} w_{h}^{-2}\right) \times\left(\sum_{i=0}^{+\infty} \frac{\left|y_{1+i}\right|^{2}}{\left\|\gamma_{1+i}\right\|_{t, \beta}^{2}}\right)$, so the majorisation is of interest only when the series $\sum_{h=1}^{+\infty} w_{h}^{-2}$ is convergent, which we shall assume until a later more precise hypothesis.

We rewrite

$$
A=\sum_{h=1}^{+\infty} \sum_{i=0}^{+\infty} \tilde{A}(h, i) \frac{\left|y_{2 h+1+i}\right|^{2}}{\left\|\gamma_{2 h+1+i}\right\|_{t, \beta}^{2}}
$$

and

$$
B=\sum_{h=1}^{+\infty} \sum_{i=0}^{+\infty} \tilde{B}(h, i) \frac{\left|y_{2 h+2+i}\right|^{2}}{\left\|\gamma_{2 h+2+i}\right\|_{t, \beta}^{2}}
$$

with

$$
\tilde{A}(h, i)=A(h, i)^{2} w_{h}^{2}\left\|\gamma_{i+1}\right\|_{t, \beta}^{2}\left\|\gamma_{2 h+1+i}\right\|_{t, \beta}^{2}
$$

and

$$
\tilde{B}(h, i)=B(h, i)^{2} w_{h}^{2}\left\|\gamma_{i+1}\right\|_{t, \beta}^{2}\left\|\gamma_{2 h+2+i}\right\|_{t, \beta}^{2} .
$$

We shall prove that the condition $\alpha \leqslant 1$ and some conditions on the relative values of $\alpha, \beta$ and $t$ are sufficient for $\tilde{A}(h, i)$ and $\tilde{B}(h, i)$ to be majorised, uniformly in $i$, by the general term of a convergent series in $h$, respectively $a_{h}$ and $b_{h}$. Then $A$ will be majorised by

$$
\sum_{h=1}^{+\infty} \sum_{i=0}^{+\infty} a_{h} \frac{\left|y_{2 h+1+i}\right|^{2}}{\left\|\gamma_{2 h+1+i}\right\|_{t, \beta}^{2}}=\sum_{h=1}^{+\infty} a_{h} \sum_{i=0}^{+\infty} \frac{\left|y_{2 h+1+i}\right|^{2}}{\left\|\gamma_{2 h+1+i}\right\|_{t, \beta}^{2}} .
$$

Since for any $h \geqslant 1$,

$$
\sum_{i=0}^{+\infty} \frac{\left|y_{2 h+1+i}\right|^{2}}{\left\|\gamma_{2 h+1+i}\right\|_{t, \beta}^{2}} \leqslant \sum_{s=1}^{+\infty} \frac{\left|y_{s}\right|^{2}}{\left\|\gamma_{s}\right\|_{t, \beta}^{2}},
$$

there will exist a constant $C_{A}>0$, independent of $h$, such that

$$
A \leqslant C_{A} \sum_{s=1}^{+\infty} \frac{\left|y_{s}\right|^{2}}{\left\|\gamma_{s}\right\|_{t, \beta}^{2}},
$$


as we may take, e.g., $C_{A}=\sum_{h=0}^{+\infty} a_{h}$. A similar argument shows that there will exist a constant $C_{B}>0$ independent of $h$ such that

$$
B \leqslant C_{B} \sum_{s=1}^{+\infty} \frac{\left|y_{s}\right|^{2}}{\left\|\gamma_{s}\right\|_{t, \beta}^{2}}
$$

End of the proof. Writing down the expressions for $\tilde{A}(h, i)$ and $\tilde{B}(h, i)$ and applying repeatedly analogous majorisations as above, we obtain that there exists a constant $c$ independant of $h$ and $i$ such that $\tilde{A}(h, i)$ and $\tilde{B}(h, i)$ are both majorised by $c \cdot W^{\prime}(h) W^{\prime \prime}(i)$ where

$$
\begin{aligned}
W^{\prime}(h) & =\frac{w_{h}^{2}}{h} t^{-\frac{4 h}{\beta}} \frac{\Gamma(8 h) \Gamma\left(\frac{16 h}{\beta}\right)^{\frac{1}{4}}}{\Gamma(2 h)^{2} \Gamma\left(\frac{4 h}{\alpha}\right)^{2}}, \\
W^{\prime \prime}(i) & =\frac{t^{-\frac{4 i}{\beta}} \Gamma\left(\frac{2}{\beta}(i+2)\right) \Gamma(4(i+1)) \Gamma\left(\frac{4}{\beta}(i+1)\right)^{\frac{1}{4}}}{(1+i) ! 2(1+i) \Gamma\left(\frac{2}{\alpha}(i+1)\right)^{2} \Gamma(i+2)^{2}} .
\end{aligned}
$$

From now on, we fix $w_{h}=h^{\lambda}$ where $\lambda$ is any positive number such that $\sum_{h=1}^{+\infty} w_{h}^{-2}<+\infty$, i.e. $\lambda>\frac{1}{2}$. As $h \rightarrow+\infty$, we have the asymptotic relation

$$
W^{\prime}(h) \sim c \cdot h^{4\left(\frac{1}{\beta}-\frac{1}{\alpha}\right) h+4\left(1-\frac{1}{\alpha}\right) h}\left(32 t^{-\frac{1}{\beta}} \frac{\left(\frac{16}{\beta}\right)^{\frac{1}{\beta}}}{\left(\frac{16}{\alpha}\right)^{\frac{1}{\alpha}}} e^{\frac{2}{\alpha}-\frac{1}{\beta}-1}\right)^{4 h} h^{2 \lambda+\frac{3}{8}}
$$

where $c$ is a constant independent of $h$. As $i \rightarrow+\infty$, we have the equivalent

$$
W^{\prime \prime}(i) \sim c \cdot i^{4\left(\frac{1}{\beta}-\frac{1}{\alpha}\right) i}\left(\left(\frac{2}{\beta}\right)^{\frac{2}{\beta}}\left(\frac{2}{\alpha}\right)^{-\frac{1}{\alpha}} 4 e^{-\left(\frac{1}{\beta}-\frac{1}{\alpha}\right)} t^{-\frac{1}{\beta}}\right)^{4 i} i^{\frac{6}{\beta}-\frac{4}{\alpha}-\frac{25}{8}}
$$

where $c$ is a constant independent of $i$.

This proves that if one of the following conditions is satisfied

(1) $\alpha \leqslant 1, \alpha<\beta$ and $t>0$,

(2) $\alpha \leqslant 1, \alpha=\beta$ and $t>\sup \left(\frac{2}{\alpha} 4^{\alpha}, 32\right)$,

then the series of general term $W^{\prime}(h)$ is convergent and the term $W^{\prime \prime}(i)$ is bounded.

The theorem follows. 


\section{Proof of Theorem 2}

We shall now prove Theorem 2, which extends to other values of $s$ and $\sigma$ the result of Theorem 1 in the case $\alpha=\beta$.

Let $\sigma$ be any non zero complex number.

Assume $s=1$.

Following the same lines as in the proof of Theorem 1, we obtain the next result.

Proposition 7. Let $\alpha>0$ and $\sigma \in \mathbf{C}^{*}$.

(i) If $\alpha<1$, there exists $t_{1}>0$ such that for any $t>t_{1}, S_{\sigma}$ induces an unbounded, densely defined, closed and surjective operator from $H(t, \alpha)$ onto $H(1, \alpha)$.

(ii) If $|\sigma|<\sqrt{2 \pi}$, there exists $t_{2}>0$ such that for any $t>t_{2}, S_{\sigma}$ induces an unbounded, densely defined, closed and surjective operator from $H(t, 1)$ onto $H(1,1)$.

Now we obtain Theorem 2 as a consequence of the previous proposition and the next clear lemma.

Lemma 1. Let $\alpha, \beta, t$ and $s$ be real and positive. Let $\mu \in \mathbf{C}^{*}$. Let $m_{\mu}: z \mapsto \mu z$ acting on $z \in \mathbf{C}$, and $M_{\mu}: f \mapsto f \circ m_{\mu}$ acting on $f \in \mathcal{O}(\mathbf{C})$. Let $\sigma \in \mathbf{C}^{*}$.

(i) $M_{\mu}$ is a continuous one-to-one operator from $H(s, \alpha)$ to $H\left(s|\mu|^{\alpha}, \alpha\right)$.

(ii) The following diagram commutes:

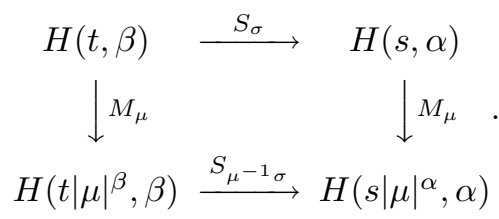

(iii) $S_{\sigma}: \operatorname{dom}\left(S_{\sigma}\right) \subset H(t, \beta) \rightarrow H(s, \alpha)$ is surjective if and only if $S_{\mu^{-1} \sigma}: \operatorname{dom}\left(S_{\mu^{-1} \sigma}\right) \subset H\left(t|\mu|^{\beta}, \beta\right) \rightarrow H\left(s|\mu|^{\alpha}, \alpha\right)$ is surjective.

\section{Further results and applications}

\subsection{Pointwise estimates for elements of $H(s, \alpha)$.}

Let $\alpha>0, s>0$. Let $f \in H(s, \alpha)$. For any $z \in \mathbf{C}$ and any $r>0$, the mean-value property and the Cauchy-Schwarz inequality give

$$
|f(z)|^{2} \leqslant C(s, \alpha, r, z) \int_{D(z, r)}|f(w)|^{2} e^{-s|w|^{\alpha}} d \lambda(w)
$$

where $D(z, r)$ is the disk of center $z$ and radius $r$, and $C(s, \alpha, r, z)$ a positive constant depending only on $s, \alpha, r$ and $z$. 
Minimizing $C(s, \alpha, r, z)$ with respect to $r$, we obtain [3] two positive constants $C$ and $M$ such that

$$
|f(z)| \leqslant C \cdot\|f\|_{s, \alpha} \cdot|z|^{\alpha-1} e^{\frac{s}{2}|z|^{\alpha}}
$$

for all $f \in H(s, \alpha)$ and $|z| \geqslant M$.

Applying the results of $[\mathbf{1 4}]$ and $[\mathbf{9}$, Lemma 18(a)], we obtain a positive constant $C$ such that

$$
|f(z)| \leqslant C \cdot\|f\|_{s, \alpha} \cdot|z|^{\frac{\alpha}{2}-1} e^{\frac{s}{2}|z|^{\alpha}}
$$

for all $f \in H(s, \alpha)$ and $z \in \mathbf{C}^{*}$.

For a more general statement in $L^{p}$-spaces, see [3]. For analogous statements in Bargmann-Fock spaces, see [15] and [11].

\subsection{Periodic functions.}

If $g$ vanishes identically then a solution $f$ of (1.1) is a $\sigma$-periodic function.

Proposition 8. Let $\sigma$ be a non-zero complex number and $f \in H(s, \alpha)$ be $\sigma$-periodic.

(i) If $\alpha<1$ then $f=c \in \mathbf{C}$, a constant.

(ii) If $\alpha=1$ then there exists an $N \in \mathbf{N}$ such that for any $z \in \mathbf{C}$, $f(z)=\sum_{k=-N}^{+N} c_{k} e^{2 i \pi k \sigma^{-1} z}$ with $c_{k} \in \mathbf{C}$ for $-N \leqslant k \leqslant N$. If moreover $s|\sigma|<4 \pi$ then $f$ is a constant.

Proof: (Inspired by Markushevich [10, Th 4.10, t III, Ch 4].) Since $f$ is $\sigma$-periodic, we may write $f(z)=\sum_{k=-\infty}^{+\infty} c_{k} e^{2 i k \pi \sigma^{-1} z}$ for $z$ in $\mathbf{C}$, where the coefficients $c_{k}$ are given by

$$
c_{k}=\frac{1}{2 \pi r^{k}} \int_{0}^{2 \pi} f\left(\frac{\sigma}{2 \pi}(\theta-i \ln r)\right) e^{-i k \theta} d \theta
$$

for any $k \in \mathbf{Z}$ and any $r>0$. From (7.2) and (7.1) we deduce

$$
\left|c_{k}\right| \leqslant \frac{C\|f\|}{2 \pi r^{k}} \int_{0}^{2 \pi} e^{\frac{s}{2} \frac{\mid \sigma \sigma^{\alpha}}{(2 \pi)^{\alpha}}\left(\theta^{2}+(\ln r)^{2}\right)^{\frac{\alpha}{2}}} d \theta
$$

and so

$$
\left|c_{k}\right| \leqslant \frac{C\|f\|}{r^{k}} e^{\frac{s}{2} \frac{|\sigma|^{\alpha}}{(2 \pi)^{\alpha}}\left((2 \pi)^{2}+(\ln r)^{2}\right)^{\frac{\alpha}{2}}},
$$

where $C$ is a constant. 
Consider first $\alpha<1$. Letting $r \rightarrow 0$ in (7.3) we see that $c_{k}=0$ for $k \leqslant-1$, while letting $r \rightarrow+\infty$ we see that $c_{k}=0$ for $k \geqslant 1$. This proves that $f=c_{0}$, a constant.

Consider $\alpha=1$. Letting $r \rightarrow 0$ in (7.3) we see that $c_{k}=0$ for $k<-\frac{s}{2} \frac{|\sigma|}{2 \pi}$ while letting $r \rightarrow+\infty$ we see that $c_{k}=0$ for $k>\frac{s}{2} \frac{|\sigma|}{2 \pi}$.

The proposition follows.

\subsection{An application.}

From Proposition 7.1 and Theorem 2 we obtain the following theorem.

Theorem 3. Let $\sigma \in \mathbf{C}^{*}$. Let $\alpha \leqslant 1$ and $s>0$.

(1) If $\alpha<1$, there exists $t^{\prime}>0$ such that:

For any $t>t^{\prime}$ and any entire function $g$ verifying

$$
g(z)=O\left(e^{s|z|^{\alpha}}\right)
$$

as $|z| \rightarrow+\infty$, there exists an entire function $f$ verifying

$$
\begin{aligned}
& f(z+\sigma)-f(z)=g(z) \quad \text { for any } z \in \mathbf{C}, \\
& f(z)=O\left(|z|^{\frac{\alpha}{2}-1} e^{t|z|^{\alpha}}\right) \quad \text { as }|z| \rightarrow+\infty .
\end{aligned}
$$

(2) If $s|\sigma|<\sqrt{2 \pi}$, there exists $t^{\prime \prime}>0$ such that:

For any $t>t^{\prime \prime}$ and any entire function $g$ verifying

$$
g(z)=O\left(e^{s|z|}\right)
$$

as $|z| \rightarrow+\infty$, there exists an entire function $f$ verifying

$$
f(z+\sigma)-f(z)=g(z) \quad \text { for any } z \in \mathbf{C},
$$

$$
f(z)=O\left(|z|^{-\frac{1}{2}} e^{t|z|}\right) \quad \text { as }|z| \rightarrow+\infty .
$$

Acknowledgements. The author wishes to thank the referee who suggested the estimate (7.1) to him and Niklas Lindholm for helpful advice during the Anacoga-Conference in Wuppertal in July 2002.

\section{References}

[1] C. A. Berenstein And R. Gay, "Complex analysis and special topics in harmonic analysis", Springer-Verlag, New York, 1995.

[2] B. BRIVE, Somme d'une fonction entière de certains espaces $L^{2}$ à poids, C. R. Acad. Sci. Paris Sér. I Math. 330(2) (2000), 83-86. 
[3] B. BRIVE, Sommes d'une fonction entière de certains espaces de type $L^{2}$ à poids, $\mathrm{PhD}$ thesis, Université de Lille (2000).

[4] B. Epstein, "Orthogonal families of analytic functions", The Macmillan Co., New York, Collier-Macmillan Ltd., London, 1965.

[5] C. Guichard, Sur la résolution de l'équation aux différences finies $g(x+1)-g(x)=h(x)$, Annales Sci.de l'École Normale 4 (1887), $361-380$.

[6] L. Hörmander, "An introduction to complex analysis in several variables", third edition, North-Holland Mathematical Library 7, North-Holland Publishing Co., Amsterdam, 1990.

[7] A. Hurwitz, Sur l'intégrale finie d'une fonction entière, Acta Math. 20 (1897), 285-312.

[8] S. G. Krantz, "Function theory of several complex variables", second edition, The Wadsworth \& Brooks/Cole Mathematics Series, Wadsworth \& Brooks/Cole Advanced Books \& Software, Pacific Grove, CA, 1992.

[9] N. Marco, X. Massaneda and J. Ortega-Cerdà, Interpolating and sampling sequences for entire functions, Preprint (2002).

[10] A. I. Markushevich, "Theory of functions of a complex variable", Vol. I, translated and edited by Richard A. Silverman, Prentice-Hall, Inc., Englewood Cliffs, N.J., 1965.

[11] X. Massaneda and P. J. Thomas, Interpolating sequences for Bargmann-Fock spaces in $\mathbb{C}^{n}$, Indag. Math. (N.S.) 11(1) (2000), $115-127$.

[12] L. M. Milne-Thomson, "The Calculus of Finite Differences", Macmillan and Co., Ltd., London, 1981.

[13] D. J. Newman and H. S. Shapiro, Certain Hilbert spaces of entire functions, Bull. Amer. Math. Soc. 72 (1966), 971-977.

[14] J. Ortega-Cerdì And K. SeIP, Beurling-type density theorems for weighted $L^{p}$ spaces of entire functions, J. Anal. Math. 75 (1998), $247-266$.

[15] K. SEIP, Density theorems for sampling and interpolation in the Bargmann-Fock space. I, J. reine angew. Math. 429 (1992), 91-106.

[16] J. M. Whittaker, "Interpolatory function theory", Cambridge Tracts in Mathematics and Mathematical Physics 33, StechertHafner, Inc., New York, 1964.

[17] K. Yosida, "Functional analysis", sixth edition, Grundlehren der Mathematischen Wissenschaften 123, Springer-Verlag, Berlin-New York, 1980. 
Laboratoire d'Arithmétique, Géométrie, Analyse et Topologie UMR CNRS 8524

UFR de Mathématiques Pures et Appliquées

Université des Sciences et Technologies de Lille

59665 Villeneuve d'Ascq cedex

France

E-mail address: brive@agat.univ-lille1.fr

Primera versió rebuda el 3 d'abril de 2002, darrera versió rebuda el 18 de juliol de 2002. 